\title{
Home Alone: Assessing Mobility Independence Before Discharge
}

Dennis M. Manning, MD, FACP, FACC ${ }^{1}$

A. Scott Keller, $\mathrm{MD}^{2}$

Debra L. Frank, RN, MSN ${ }^{1}$

\author{
${ }^{1}$ Department of Medicine, Mercy Hospital of Pittsburgh, Pittsburgh, Pennsylvania. \\ ${ }^{2}$ Division of Hospital Internal Medicine, Mayo Clinic, Rochester, Minnesota.
}

D.M.M. is now with the Division of Hospital Internal Medicine, Mayo Clinic, Rochester, Minnesota.

Supported by the National Center for Research Resources (NCRR), a component of the National Institutes of Health (NIH), and the NIH Roadmap for Medical Research (1 UL1 RR024150*). The contents of this work are solely the responsibility of the authors and do not necessarily represent the official view of NCRR or NIH. Information on NCRR is available at http://www.ncrr.nih.gov. Information on Reengineering the Clinical Research Enterprise can be obtained from http://nihroadmap.nih.gov.

Presented as a poster at the 5th Annual Meeting of the National Association of Inpatient Physicians, Philadelphia, Pennsylvania, April 9, 2002.

Disclosure: Nothing to report.

Hospitalists are often confronted with discharge planning responsibility and decisions for elderly patients who live alone. The absence of an in-home helper (spouse, partner, or care-giver) reduces the margin of safety and resilience to any new debility. Research has documented that during hospital stays elderly patients tend to become deconditioned, even if there is no new specific neurologic or motor deficit. In the patient whose pre-hospital mobility independence is not robust, and perhaps marginally compensated, inpatient stays for any diagnosis may result in critical decrements in mobility independence. The present study is an effort to design a bedside tool for the hospitalist by which to discern, or screen, for such debility. The tool is a hierarchical performance test we named I-MOVE (Independent Mobility Validation Examination). It is a quick series of bedside mobility requests to demonstrate capability of fundamental movements critical to independent living. We describe manner in which I-MOVE can be performed. Moreover, we describe the face validity and the high interrater reliability ( $>0.90$ intra-class correlation coefficient) of two RNs who independently administered and scored I-MOVE for 41 patients on a General Medical Care Unit. Although not yet studied in correlation with outcomes, nor with validated mobility assessment tools, we believe I-MOVE can serve as a useful extension of the nurse's assessment, or the Hospitalist's physical examination. Discerning the continued capability of mobility independence is a desirable, on-going insight for discharge planning of the elderly patient who resides alone. Journal of Hospital Medicine 2009;4:252-254. (C) 2009 Society of Hospital Medicine.

KEYWORDS: discharge planning, geriatric assessment, hospital care, mobility.

Hospitalized patients are often debilitated, either from their admitting illness or from the deconditioning that occurs with inactivity. Functional decline, which appears to progress in a hierarchical pattern, ${ }^{1}$ occurs in $24 \%$ to $50 \%$ of geriatric patients during hospitalization and is poorly documented. ${ }^{2}$ Such a decline is associated not only with longer hospital stays and increased health care costs but also with higher mortality. ${ }^{3}$ The American College of Physicians, through its "Assessing Care of Vulnerable Elders" project, expressly endorsed "gait and mobility evaluation" as a quality indicator, and examination insufficiency is well documented. ${ }^{4}$

Of the several existing mobility assessment tools, few are used routinely in hospital. Some require complex scoring; others require timing and/or a trained occupational therapist. ${ }^{5}$ We created a simplified tool named "Independent Mobility Validation Examination" (I-MOVE) for use by bedside caregivers. We evaluated the tool's face validity and interobserver agreement.

\section{I-MOVE}

I-MOVE, represented schematically in Figure 1 , is a performance test that assesses the patient's ability to perform a sequence of 6 basic tasks: rolling over in bed, sitting up, standing, transferring to a chair, walking in the room, and walking in the hallway. Most motor functions can be assumed to be hierarchical in nature; any patient who can perform at the highest level, such as walking safely, also would be expected to perform at the lowest level.

Instructions for administering I-MOVE are as follows:

1. Review current orders. Exclude patients ordered on "bed rest" or "non-weight-bearing" or other orders precluding any of the 6 requested actions. 


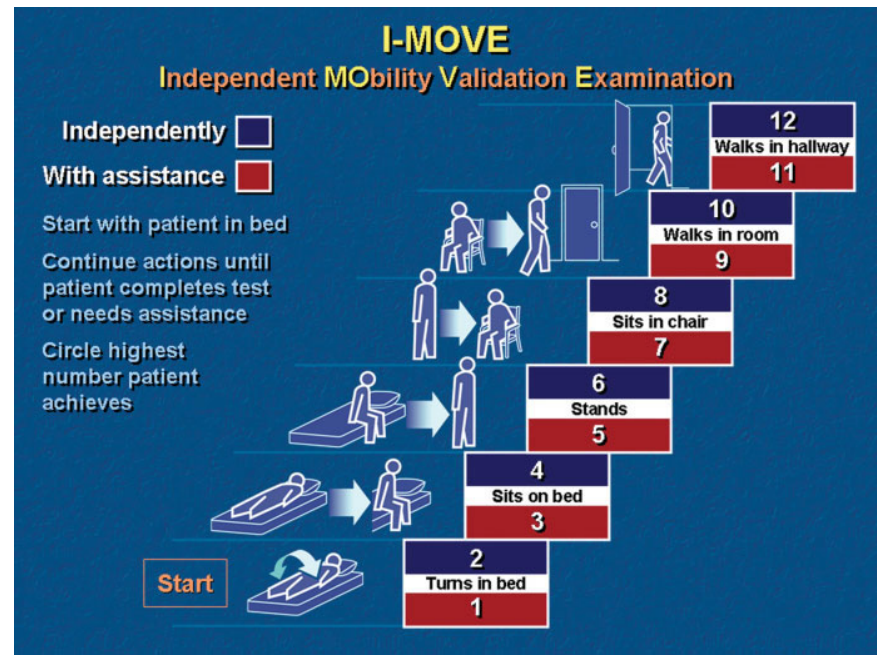

FIGURE 1. Schematic diagram of requested movements and scoring.

2. Prepare environment.

a. Chair at bedside.

b. Lower side bed rail closest to chair.

c. Clear path for patient to ambulate.

d. Ensure patient dons slippers.

e. Flatten bed.

f. Ensure any gait assistive device, if generally used by the patient, is within reach from the bedside.

3. Requests for patient action (for steps $\mathrm{c}$ through $\mathrm{f}$, make available and within reach any appropriate gait-assistance device such as walker or cane, if such is customarily used at home or newly prescribed):

a. With patient lying supine in bed, with close supervision, ask patient to turn from side to side in bed (request when both bed rails are up).

b. Lower side rail closer to chair and ask the patient to rise up to a sitting position and turn to sit up with legs dangling off the bed.

c. Ask the patient to stand.

d. Ask the patient to take a seat in the chair next to the bed.

e. Ask the patient to ambulate in the room.

f. Ask the patient to ambulate in the hallway.

4. At any point if the patient seems incapable, unsteady, or unsafe to accomplish the requested task, render handson assistance and immediately end the test.

5. Document, by number (1-12), the activity level successfully accomplished independently by the patient (even number levels) or accomplished with assistance (odd number levels).

6. Patient may be considered independent if able to perform the activity with a normal assistive device (cane, walker, brace, or crutches) but not using furniture.

7. Assistance is defined as any physical contact with the patient.

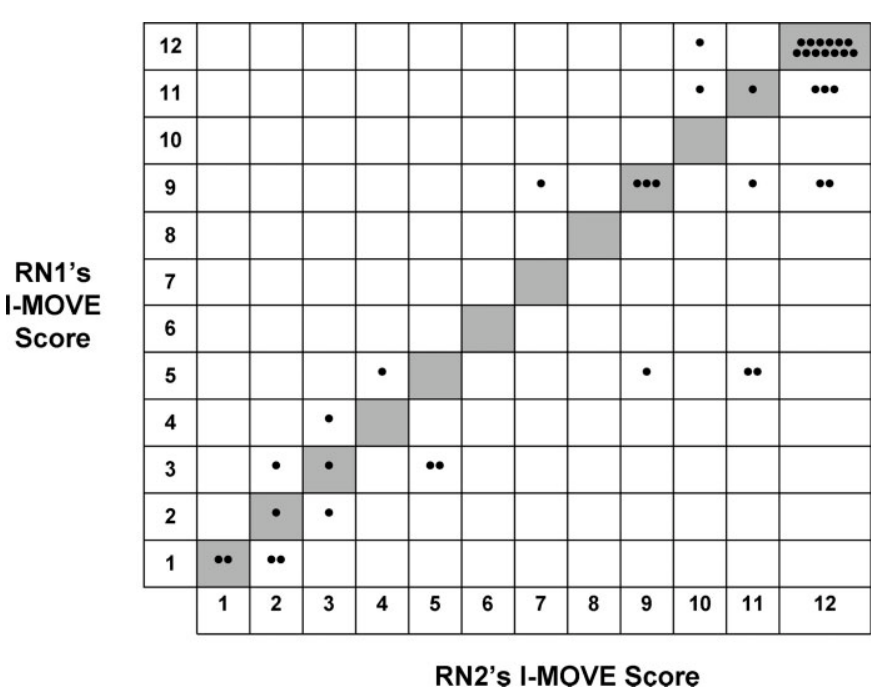

FIGURE 2. Interrater reliability. Each dot represents 1 patient's pair of I-MOVE scores evaluated independently by RN1 and RN2 within 1 hour's time.

\section{Findings}

\section{Face Validity}

We sent surveys to 6 experienced practicing clinicians at our hospital: a geriatrician, a physiatrist, an exercise physiologist, an occupational therapist, a physical therapist, and a registered nurse. We asked each clinician to rate the 6 IMOVE elements (requested actions) for clinical relevance to mobility independence. Relevance of each element was measured on an ordinal scale with scores ranging from 1 to 4, with: 1 "not relevant"; 2 "somewhat relevant"; 3 "quite relevant"; and 4 "very relevant". From the 5 responses we received, 4 evaluators ranked all 6 I-MOVE requested actions as "very relevant". The fifth evaluator ranked 5 of the 6 actions as "very relevant" and 1 action (walking in the room) as "quite relevant". These results demonstrate general agreement that I-MOVE is, at face value, a reasonable measure of independent mobility.

\section{Interrater Reliability}

The protocol was approved by the hospital's institutional review board. On a general medical unit-a non-electrocardiographic telemetry, nonsurgical unit of an acute care hospital, where patients are assigned the primary service of an internal medicine physician-we instructed 2 registered nurse (RN) volunteers (RN1 and RN2) in the I-MOVE protocol. Each RN administered I-MOVE independently to 41 consecutive, cognitively intact patients in a blinded fashion (ie, neither nurse was aware of the other's scoring of each patient) and within 1 hour of each other's assessment.

After administering I-MOVE to each patient, the nurse judged and scored the patient's performance using the 12level I-MOVE ordinal scale, ranging from a low value of 1 , 
complete dependence, to the highest value of 12 , complete independence. The patients' I-MOVE score pairs recorded by RN1 and RN2 were statistically compared. Interrater reliability, a comparison of the 41 patients' score pairs, is graphically represented in Figure 2. The calculated intraclass correlation coefficient $(r)$ was 0.90 , indicating "excellent" agreement $(r>0.75)$.

\section{Discussion}

Traditional physical examinations by physicians and assessments by nurses do not routinely extend to standardized mobility testing and may fail to recognize disability. Of the existing mobility assessment tools, we believe that most are not suited to patients hospitalized on general medical units. I-MOVE has been designed to address this need, with an emphasis on practicality and brevity to allow repetition at appropriate intervals ("tracking"), as is done for vital signs. In this initial study, I-MOVE was found to have face-valid content and excellent interrater agreement.

Our study had several limitations. Only 1 pair of test administrators was involved; the sample population was chosen by convenience; clustering of outcomes occurred at level 12, which may have augmented the agreement; and the study was limited to cognitively intact patients. Note that we chose to use the intraclass correlation coefficient rather than the $\kappa$ statistic because the weighting between the ordinal I-MOVE scores has not yet been studied and defined. Also, the weighted $\kappa$ is asymptotically equivalent to the intraclass correlation coefficient.

I-MOVE is intended to aid caregivers in the recognition of debility so that appropriate interventions such as physi- cal therapy may be prescribed. It was designed to complement, not replace, specialized evaluations such as those performed by physical therapists, occupational therapists, or comprehensive geriatric assessments. This practical assessment of basic functioning may enhance communication among caregivers, patients, and patients' family members, especially with regard to discharge planning. Further study is needed to validate I-MOVE against existing tools, evaluate I-MOVE's utility as a "vital sign," and discern whether a sharp or unexpected decline portends a medical complication.

\section{Address for correspondence and reprint requests:}

Dennis M. Manning, MD, Division of Hospital Internal Medicine, Mayo Clinic, 200 First Street SW, Rochester, MN 55905;

Telephone: 507-284-8917; Fax: 507-284-2107;

E-mail: manning.dennis@mayo.edu Received 29 August 2005; revision received 7 July 2008; accepted 22 September 2008.

\section{References}

1. Gerely MB. Health status and physical capacity. In: Osterweil D, BrummelSmith K, Beck JC, eds. Comprehensive Geriatric Assessment. New York: McGraw-Hill; 2000:41-66.

2. Inouye SK, Wagner DR, Acampora D, et al. A predictive index for functional decline in hospitalized elderly medical patients. J Gen Intern Med. 1993;8(12):645-652.

3. Brown CJ, Friedkin RJ, Inouye SK. Prevalence and outcomes of low mobility in hospitalized older patients. J Am Geriatr Soc. 2004;52(8):1263-1270.

4. Rubenstein LZ, Solomon DH, Roth CP, et al. Detection and management of falls and instability in vulnerable elders by community physicians. J Am Geriatr Soc. 2004;52(9):1527-1531.

5. Mudge AM, Giebel AJ, Cutler AJ. Exercising body and mind: an integrated approach to functional independence in hospitalized older people. J Am Geriatr Soc. 2008;56(4):630-635. 